\title{
Identification with Face Recognition Methods in Real Life Applications
}

\author{
Çağla Ediz* \\ * Department of Management Information Systems, Sakarya University, Sakarya, Turkey \\ (cediz@sakarya.edu.tr)
}

Received: 30.10.2020 Accepted: 18.07.2021

\begin{abstract}
The development of technologies such as employment tracking systems, personal security, and the use of robots has led a lot of studies on face recognition systems. In the most of studies considering face recognition, recognition accuracies are very high, since training and testing images are selected randomly from the same databases. However, in real life applications, these images are not randomly selected from the same database. Because, these systems are trained during installation of the recognition system or when a new person needs to be introduced to the system. On the other hand, images used for predictions are uploaded to the system at other times. In this study, it is aimed to show that the accuracy rates of real-life face recognition systems differ from the systems trained and tested with randomly selected images as usually done in literature. To observe this difference in the first step, training and test images are selected randomly. In the second step, training and test images are divided according to the recording dates as in real life. Accuracy rates are evaluated by using linear discriminant analysis, local binary patterns and principal component analysis methods. Although the accuracies are very high for the first step, it is seen that the accuracies fell dramatically in the second step for all methods. Afterwards a new method is searched also in this study to increase these low accuracy rates. It is shown that usage of eye area images instead of face images has higher accuracy rates in all above methods for real life applications.
\end{abstract}

Keywords: Eye Area Recognition, Face Recognition, Image Processing, Linear Discriminant Analysis, Local Binary Patterns, Principal Component Analysis, OpenCV

\section{Introduction}

Face recognition systems are generally considered in two groups as feature-based methods and holistic approach methods. In feature-based methods, the face to be recognized is compared with other faces in the database by using certain geometric features [1]. In holistic methods, machine learning and deep learning techniques are used. The system continues to learn with the fed digital images and uses the learned features while comparing the newly encountered images with those in the database. Although different methods have been developed in face recognition systems, it is still very hard to get 100\% accurate results from these systems [2]. The current methods have some advantages and disadvantages. While training times of some holistic approach methods, such as deep learning, take more time, training times of feature-based methods are generally take short time. On the other hand, accurate prediction rates of holistic approach methods are higher than feature based methods.

In the studies considering face recognition, databases offering ready face images are frequently used. There are many databases created for using in face recognition research, such as Yale database, AR Face database, CAS-PEAL database, FERET database, XM2VTS database, PIE database, and KFDB [3].

Considering the existing literature on face recognition, in a study using a ready database with face images, OpenCV and Dlib libraries, which are popular in face recognition systems, are compared, and OpenCV's performance is found to be higher [4]. In another study, the faces in the MOBIO database obtained by using mobile devices are compared with the face recognition systems used in OpenCV and the best performances are obtained with LDA in high image sizes and with LBPH in low image sizes [5]. In their study, Abdülsamed and Olcay observed that, using the AT\&T database in facial recognition, successful results were achieved. However while they used the actor images in the movies, an average success was achieved [6]. They attributed the high recognition rate in the ready database to the constant positioning and lighting environment. On the other hand, images captured from daily life obtains low rate recognition rates because of reasons like very different light levels, different perspectives and applying 
different makeup to people at different times ([6], [7]). In Aksoy's study [8], front, side, upper and lower face images were taken from volunteer students and faculty members. The best results were obtained with PCA and SVD algorithm in the front and side face recognition system, and with the LBP algorithm in the upper and lower face recognition systems. Also, different algorithms were developed for face recognition systems in literature and the success of these algorithms in face recognition was evaluated by using known face databases such as Yale Database [9]. In recent researches, deep learning also are used to detect faces in face recognition systems [10].

It can be estimated that if training and testing images are obtained on different dates like in real life applications, the recognition systems' accuracy rates will be decreased. To investigate this estimation, this study is prepared. First of all, some recognizer methods are used to see the results. In the next section, selected face recognizer methods are investigated. Afterwards, in Section III, recognition systems' prediction rates are compared for randomly selected face images and different date recorded face images. In Section IV, a new method is applied to increase recognition accuracy rates.

\section{Selected Face Recognizer Methods}

In this article, holistic approach face recognition methods based on statistical calculations are used. These are the methods in OpenCv library called as Eigenface, Fisherface and Local Binary Patterns Histograms (LBPH). Among these methods, Eigenface and Fisherface recognizers make a face prediction based on machine learning with a holistic approach, while $\mathrm{LBPH}$ recognizer makes face prediction using local differences.

In Eigenface recognizer and Fisherface recognizer methods, the dimension number of the gray scale image vector equal to the pixels' number is reduced to a reasonable level. Then image vectors with reduced dimensions are used in trainings and predictions. The Eigenface recognizer method uses the difference of faces to the average face image. Fisherface recognizer, on the other hand, uses the average face images on faces classified by labels instead of average image of all faces. Thus, Eigenface recognizer classifies images using the Principle Component Analysis (PCA) method [11] and Fisherface recognizer classifies images using Linear Discriminant Analysis (LDA) method [12]. While PCA tries to maximize the distance between all variables, LDA tries to maximize the distance between classes [13]. Although LDA performs better than PCA except for low test numbers, it has difficulty in processing high-dimensional image data ([14], [15]).

In both methods, covariance matrices are calculated and eigenvalues and eigenvectors of covariance matrices are found. The number of dimensions in an image that is equal to the number of pixels is reduced to used image numbers. By selecting eigenvectors with high eigenvalue, the number of data can be further reduced. Afterwards, weights of trained images on eigenvectors are calculated. When an unknown image comes, the distances of this image from each trained images are calculated and the image is predicted by a classification method such as the nearest neighbor.

As the third method, LBPH face recognizer technique uses local differences in the image. In this technique, image pixels are divided into squares in certain size. Perimeter square pixels get 1 or 0 depending on whether they are larger or smaller than the middle pixel value. Perimeter square values are written in binary value by starting from the upper left corner and progressing clockwise, and then the obtained value is converted to decimal value and assigned to the central square value. By creating a histogram with the obtained values, the histogram values of the images are compared [16]. Since the face recognition system based on the LBPH technique makes local comparisons, it is less affected by the light level differences on the face images than the Eigenface and Fisherface techniques.

\section{Comparing Prediction Rates of Randomly Selected Face Images with Different Date Recorded Face Images}

In this study, images, captured at 0.2 second intervals from the videos, were used for face detection with haar cascade algorithms which are widely used in finding the facial region. These algorithms, which are presented by Viola and Jones (2001) and used in face detection, search whether the black and white areas in different combinations are present in the image [17]. The haar cascade classifiers make face detection faster than the previous face detection systems. In this study, different videos of 8 people were used to generate image data. In order to detect face images, face haar cascade algorithm is used. Some face images of the same child (Person ID 2) attained by using face haar cascade algorithm can be seen in Figure1. 


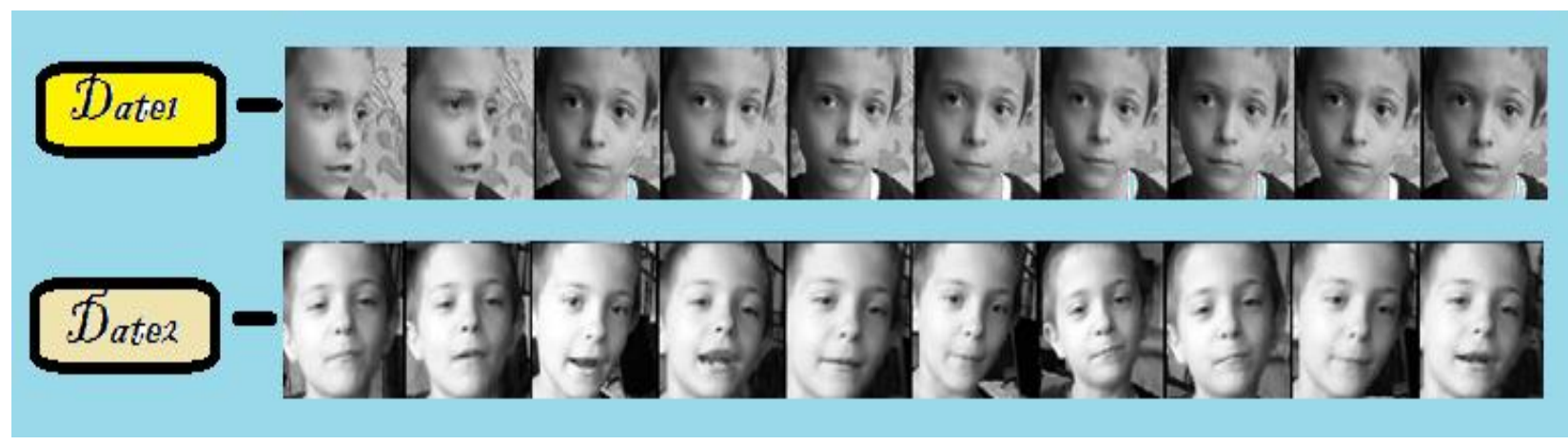

Figure 1: Some face images of the same child (Person ID 2) captured at different dates from the prepared database.

Videos recorded at different times for each person were used in the study. In the first step, images obtained from different date records were mixed randomly and some of these images were used for education and some of them were used for testing. The results of this step can be seen in Figure 2. The accuracy rates of randomly selected images for PCA, LDA and

\begin{tabular}{|c|c|c|c|c|c|c|c|c|c|c|}
\hline \multirow{2}{*}{ PersonID } & \multicolumn{3}{|c|}{ PCA } & \multicolumn{3}{|c|}{ LDA } & \multicolumn{3}{|c|}{ LBPH } & \multirow{2}{*}{ Total } \\
\hline & True & False & Not Found & True & False & Not Found & True & False & Not Found & \\
\hline 1 & 59 & & 10 & 62 & 7 & & 61 & & 8 & 69 \\
\hline 2 & 30 & & 46 & 42 & 34 & & 55 & & 21 & 76 \\
\hline 3 & 46 & & 13 & 39 & 20 & & 46 & 4 & 9 & 59 \\
\hline 4 & 56 & & & 53 & 3 & & 56 & & & 56 \\
\hline 5 & 34 & & 10 & 35 & 9 & & 44 & & & 44 \\
\hline 6 & 55 & & & 55 & & & 55 & & & 55 \\
\hline 7 & 38 & & 16 & 49 & 5 & & 51 & & 3 & 54 \\
\hline 8 & 45 & & 5 & 50 & & & 50 & & & 50 \\
\hline \multirow[t]{2}{*}{ Total } & 363 & 0 & 100 & 385 & 78 & & 418 & 4 & 41 & 463 \\
\hline & \multicolumn{3}{|c|}{$\begin{array}{l}\text { Accuracy }(\%): 78.4 \\
\text { Error }(\%): 0 \\
\text { Not Found }(\%): 21.6\end{array}$} & \multicolumn{3}{|c|}{$\begin{array}{l}\text { Accuracy }(\%): 83.2 \\
\text { Error }(\%): 16.8 \\
\text { Not Found }(\%): 0\end{array}$} & \multicolumn{3}{|c|}{$\begin{array}{l}\text { Accuracy }(\%): 90.3 \\
\text { Error }(\%): 0.9 \\
\text { Not Found }(\%): 8.9\end{array}$} & \\
\hline
\end{tabular}

Figure 2: Prediction results of randomly mixed face images used in both train and test

In the second step, some of the videos taken on different dates were used for training and some for testing. The number of training images (383) and the number of test images (463) for both stages (first and second stages) were prepared to be the same. After these preparations, the accuracy rates of face
LBPH are $78.4 ; 83.2 ; 90.3$ sequentially. LBPH attained the highest accuracy. The worst results are taken from the child whose PersonID is equal to 2 .

\begin{tabular}{|c|c|c|c|c|c|c|c|c|c|c|}
\hline \multirow{2}{*}{ PersonID } & \multicolumn{3}{|c|}{ PCA } & \multicolumn{3}{|c|}{ LDA } & \multicolumn{3}{|c|}{ LBPH } & \multirow{2}{*}{ Total } \\
\hline & True & False & Not Found & True & False & Not Found & True & False & Not Found & \\
\hline 1 & 13 & & 56 & 41 & 28 & & 41 & & 28 & 69 \\
\hline 2 & & & 76 & 10 & 66 & & & 3 & 73 & 76 \\
\hline 3 & & 2 & 57 & 11 & 48 & & 12 & 33 & 14 & 59 \\
\hline 4 & & & 56 & 41 & 15 & & 48 & 7 & 1 & 56 \\
\hline 5 & & & 44 & & 44 & & & 18 & 26 & 44 \\
\hline 6 & & & 55 & & 55 & & & & 55 & 55 \\
\hline 7 & 11 & & 43 & 54 & & & 49 & & 5 & 54 \\
\hline 8 & 18 & & 32 & 50 & & & 50 & & & 50 \\
\hline \multirow[t]{2}{*}{ Total } & 42 & 2 & 419 & 207 & 256 & 0 & 200 & 61 & 202 & 463 \\
\hline & \multicolumn{3}{|c|}{$\begin{array}{l}\text { Accuracy (\%): } 9.1 \\
\text { Error (\%): } 0.4\end{array}$} & & \multicolumn{3}{|c|}{$\begin{array}{l}\text { Error }(\%): 13.2 \\
\text { Not Found (\%):43.6 }\end{array}$} & \\
\hline
\end{tabular}

Figure 3: Prediction results of test face images which have different dates from training images' dates 
When images of people taken at different times; perspective, hairstyle, facial expressions, aging, light decrease the effectiveness of recognition systems. So, because of the factors related to time and situation, the effectiveness of recognition systems is greatly reduced as seen in Figure 4. Face recognition systems used in real life will face such problems. In order to reduce these problems as much as possible, a method is developed in the next section and compared with the classic face recognition methods.

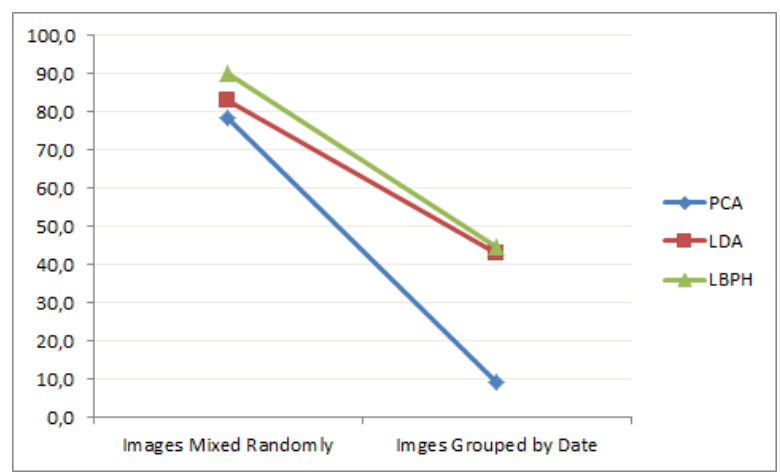

Figure 4: Accuracy rates of images mixed randomly and images grouped by date

\section{A New Method For Real Life Applications}

When trying to detect a face image using trained images taken at different dates, the accuracy rates decreased. For this reason, eye areas will be used to detect Person ID instead of faces to test the accuracy rates with the same methods.

EmguCV library derived from OpenCV is used in this study because the methods are written in Visual Studio Environment with $\mathrm{C \#}$. The steps of the algorithm to find and store the eye area images can be seen Figure 5. In these steps, first of all, face and facial images are obtained using haar cascade classifiers from training videos. Then, two eyes belonging to the same face are found. The eye area images are scaled depending on the two eye distances and rotations are made so that the lines passing through the two eye centers are on the horizontal axis. The images are converted to grayscale and a specified width and height in pixels, then they are archived in training group or test group.

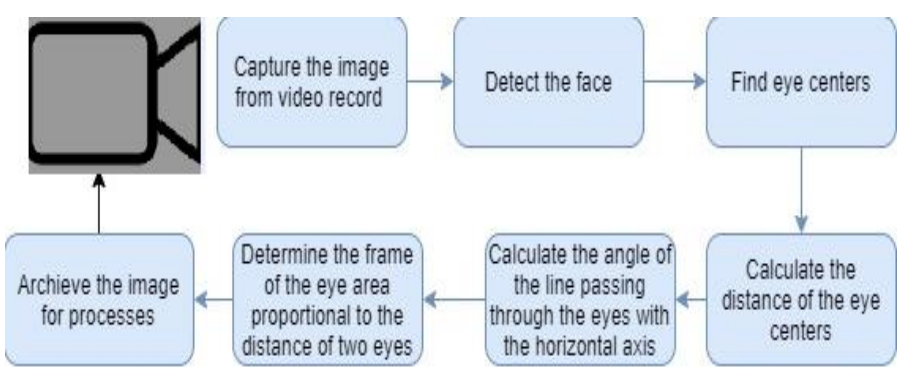

Figure 5: Algorithm steps to store the detected eye area images

Eye area images, stored in training group, are trained by using Eigenface recognizer, Fisherface recognizer and LBPH face recognizer methods. Threshold values of Eigenface recognizer, Fisherface recognizer and LBPH face recognizer methods used in the application are 4000, 2500 and 80, respectively. Then, eye area images in test group are predicted using the above methods. After predictions, the results are evaluated.

Eye haar cascade classifier and rule-based algorithms to detect the eyes belonging to the same face [18] are used in this step. However, eyes could not be detected in some of the faces in the database, since the desired conditions were not met. In approximately half of the detected face images, two eyes were found meeting the desired conditions [18]. On the faces detected eyes, the angle of the line passing through the center of the eyes was determined and the eye area image was rotated accordingly. In addition, by taking reference from two eye distances on the face images, the boundaries of the eye area images were determined and the image obtained was scaled to a certain size as seen in Figure 6.

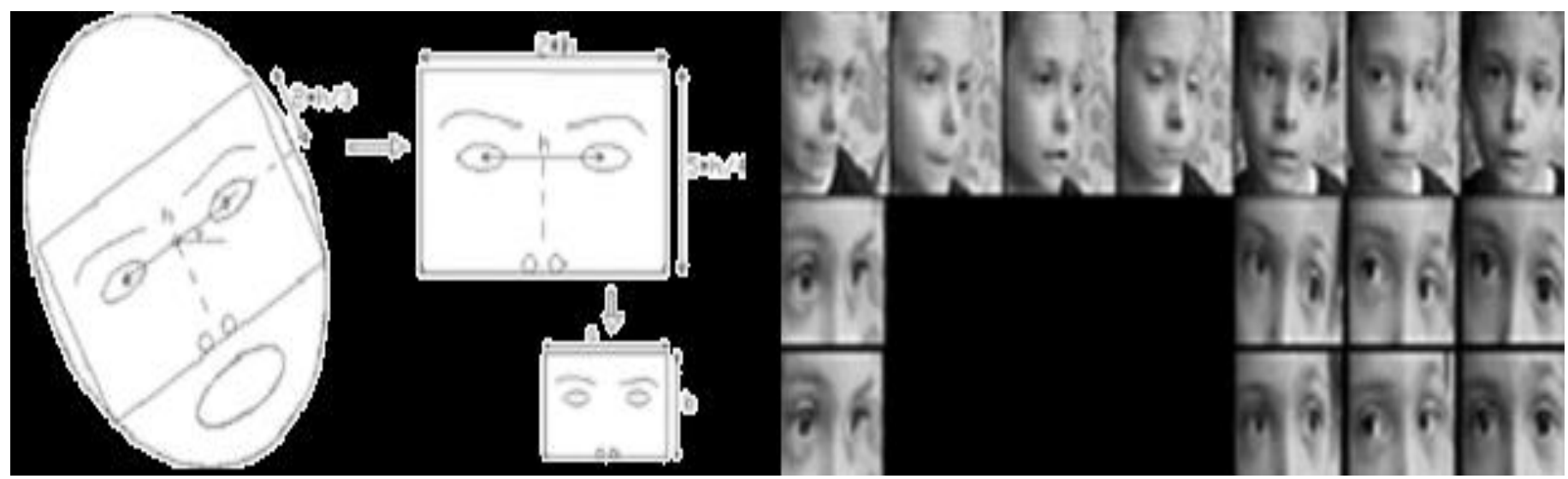

Figure 6: Trimming, rotating and resizing of eye area image 
In this study, training and test videos were recorded at different times. The eye area images (174) used in training were obtained from the archived training faces (383) and the eye regions images (208) used in the test were obtained from the archived test faces (463). Pixel sizes of all images were equalized before archiving and each image was recorded under its own group and ID number. In this step, the accuracy rates of eye area images for PCA, LDA and LBPH are 21.6; 61.5; 52.4 sequentially and LDA attained the highest accuracy as seen in Figure 7.

\begin{tabular}{|c|c|c|c|c|c|c|c|c|c|c|}
\hline \multirow{2}{*}{ Personid } & \multicolumn{3}{|c|}{ PCA } & \multicolumn{3}{|c|}{ LDA } & \multicolumn{3}{|c|}{ LBPH } & \multirow{2}{*}{ Total } \\
\hline & True & False & Not Found & True & False & Not Found & True & False & Not Found & \\
\hline 1 & 5 & & 3 & 5 & 3 & & 5 & 3 & & 8 \\
\hline 2 & & 13 & 22 & & 35 & & & 35 & & 35 \\
\hline 3 & & 29 & & 20 & 9 & & 19 & 10 & & 29 \\
\hline 4 & & 7 & 19 & 26 & & & 26 & & & 26 \\
\hline 5 & & 9 & & & 9 & & 9 & & & 9 \\
\hline 6 & 39 & 12 & & 27 & 24 & & & 51 & & 51 \\
\hline 7 & 24 & & & 24 & & & 24 & & 0 & 24 \\
\hline 8 & 25 & & 1 & 26 & & & 26 & & & 26 \\
\hline \multirow[t]{2}{*}{ Total } & 93 & 70 & 45 & 128 & 80 & 0 & 109 & 99 & 0 & 208 \\
\hline & \multicolumn{3}{|c|}{\begin{tabular}{|l} 
Accuracy $(\%): 44.7$ \\
Error $(\%): 33.7$
\end{tabular}} & \multicolumn{2}{|c|}{ Accuracy (\%):61.5 } & & $\begin{array}{l}\text { Accuracy } \\
\text { Error (\%): } \\
\text { Not Foun }\end{array}$ & 52.4 & & \\
\hline
\end{tabular}

Figure 7: Prediction results by using eye area test images which have different dates from training images' dates

In all three methods, the accuracy rates of eye area images' predictions are higher than face images' predictions for different date recorded images as seen in Figure 8. The highest accuracy rates in both face and eye area estimates are obtained with the Fisherface recognizer (LDA). However, the highest value of these accuracy rates are found to be $61.5 \%$ and still not very high.

The confusion matrices of the predictions are evaluated using the archived training and test images and the following findings areobtained. Predictions for Person ID2 are wrong totally. It is seen that the images obtained from the video used for training (14 images) and the images used for testing (35 images) have different perspectives from each other (Some images of Person ID 2 can be seen in Figure 2).

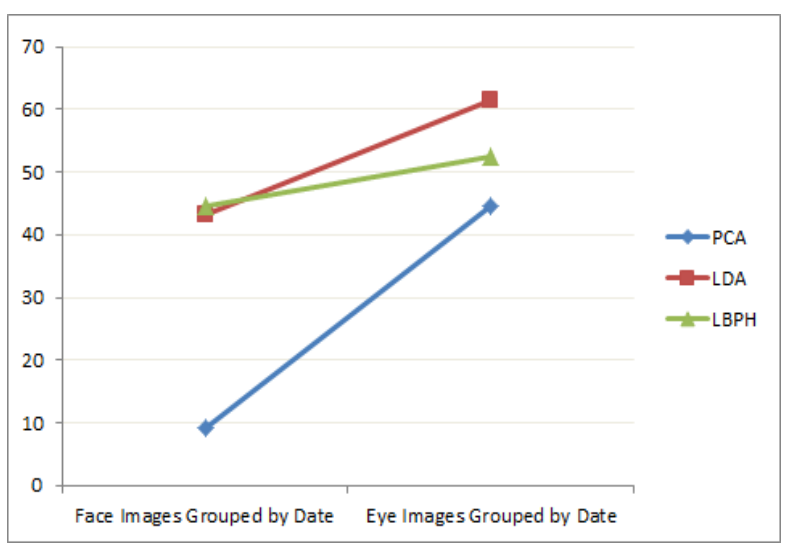

Figure 8. Accuracy rates for face and eye images when training and testing groups are separated
For Person ID 5, all of the eye area predictions, by using Eigenface and Fisherface recognizers, are false whereas all of the eye area predictions with LBPH recognizer are true, as seen in confusion matrices (Figure 9). It is noticed that average pixel values are different between training and testing images. That is, the average of pixel value of grayscale images in educational group is 112 , whereas the average of pixel for grayscale images in test group is 60 . So, it can be concluded that while PCA and LDA methods are affected by light differences, LBPH method is not affected. When histogram equalizations are performed on the same images to decrease light effect, the accuracy of the eye area images' predictions of Person ID 5 is increased. However, in this case, while total accuracy rates of Eigenface recognizer and LBPH face recognizer are increased, the Fisherface recognizer's total accuracy is rate decreased. . 


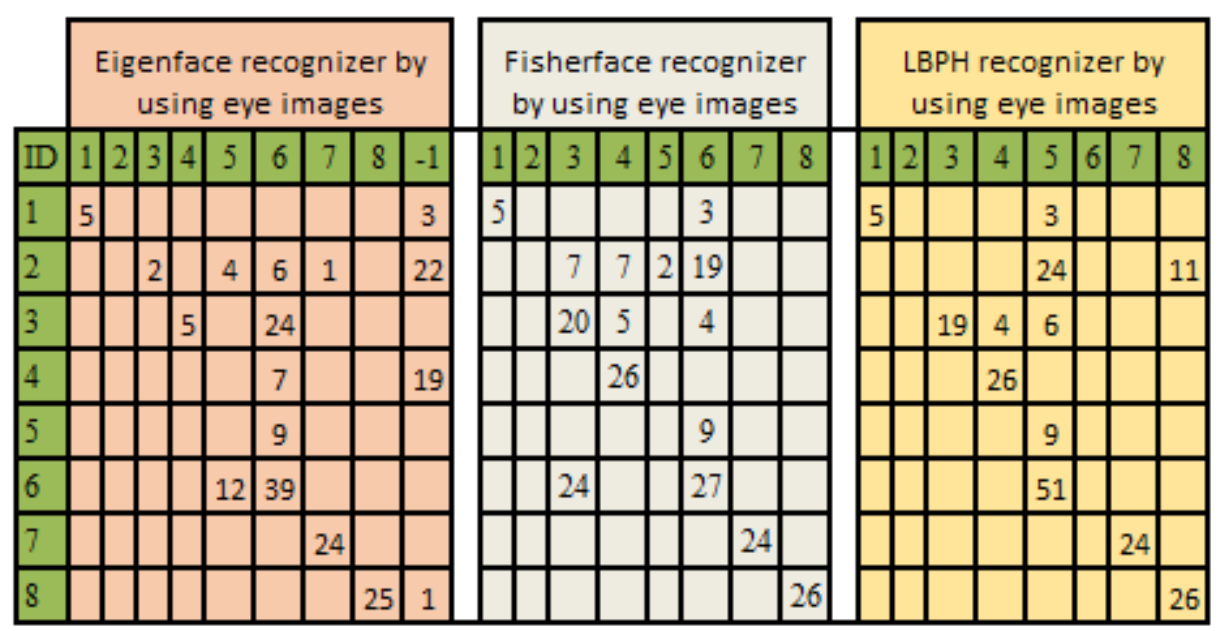

Figure 9. Confusion matrices of face recognizers for eye area images

\section{Conclusion}

In real life face recognition systems like employment tracking systems, training images' dates are different from test images' dates. However, in literature, face recognition systems are trained and tested from the same databases. It can be estimated that, the accuracy rates of literature are higher than the accuracy rates of the real life applications. To investigate this, the accuracy rates of face recognition methods trained and tested from randomly images compared with the accuracy rates of face recognition methods trained and tested from different date images are compared by using different three methods in this study. It is found that, the accuracy rates of images selected randomly are high in all methods. On the other hand, accuracy rate of trained and test from different date images is low. Therefore it can be said that, the accuracies in real life applications are not high as to be in literature. Afterwards, instead of face images, the use of eye area images is investigated in order to increase the accuracy in real-life recognition systems in this study. The eye area image prediction accuracy rates are higher than the face image estimates for all three different recognizing methods. This can be due to some changes like hair, beard or mouth opening are not affect eye area images. Also, since eye detections can be made from only the front view face images, they have a more limited positioning and this could be increased the number of accuracy predictions. In future identification systems, more specific regions of the face instead of the whole face image, can be used for identification with the help of increasing digital resolution qualities.

\section{References}

[1] Li, X. and Zhang, H. (2013, March). A survey of face recognition methods. In Proceedings of the 2nd International Conference on Computer Science and Electronics Engineering. Atlantis Press
[2] Deng, W., Hu, J., Guo, J., Cai, W. and Feng, D. (2010). Robust, accurate and efficient face recognition from a single training image: A uniform pursuit approach. Pattern Recognition, 43(5): 1748-1762.

[3] Lee, H. S., Park, S., Kang, B. N., Shin, J., Lee, J. Y., Je, H. and Kim, D. (2008, September). The POSTECH face database (PF07) and performance evaluation. In 2008 8th IEEE International Conference on Automatic Face \& Gesture Recognition (pp. 1-6). IEEE.

[4] Boyko, N., Basystiuk, O. and Shakhovska, N. (2018, August). Performance evaluation and comparison of software for face recognition, based on dlib and opencv library. In 2018 IEEE Second International Conference on Data Stream Mining \& Processing (DSMP) (pp. 478-482). IEEE.

[5] Memiş, A. and Karabiber, F. (2016, May). Face recognition on mobile environment images using appearance based methods. In 2016 24th Signal Processing and Communication Application Conference (SIU) (pp. 169-172). IEEE.

[6] Abdulsamet, H. and Olcay, T. (2017, October). Identification system from motion pictures: LBPH application. In 2017 International Conference on Computer Science and Engineering (UBMK) (pp. 845-850). IEEE.

[7] Ayata, F., Çavuş, H., İnan, M., Seyyarer, E., Biçek, E., \& Kina, E. (2020). Dostroajan: Facial Recognition Based System Input Control Agent. AJIT-e, 11(40), 82.

[8] Aksoy, B. (2019). Yüz Tanima Sistemlerinde Doğruluk Performanslarinin Değerlendirilmesi. Mühendislik Bilimleri ve Tasarım Dergisi, 7(4), 835-842.

[9] He, X., Yan, S., Hu, Y., Niyogi, P., \& Zhang, H. J. (2005). Face recognition using laplacianfaces. IEEE transactions on pattern analysis and machine intelligence, 27(3), 328-340. 
[10] Ünal, F. Z. (2019). A Comparison Of Deep Learning Based Architecture With A Conventional Approach For Face Recognition Problem. Communications Faculty of Sciences University of Ankara Series A2-A3 Physical Sciences and Engineering, 61(2), 129-149.

[11] Turk, M. and Pentland, A. (1991, January). Face recognition using eigenfaces. In Proceedings. 1991 IEEE computer society conference on computer vision and pattern recognition (pp. 586-587).

[12] Belhumeur, P. N., Hespanha, J. P. and Kriegman, D. J. (1997). Eigenfaces vs. fisherfaces: Recognition using class specific linear projection. IEEE Transactions on pattern analysis and machine intelligence, 19(7): 711-720.

[13] Kaçar, Ü., Kirci, M., Güneş, E. O. and İnan, T. (2015, May). A comparison of PCA, LDA and DCVA in ear biometrics classification using SVM. In 2015 23nd Signal Processing and Communications Applications Conference (SIU) (pp. 1260-1263). IEEE.

[14] Yang, J., Yu, H., \& Kunz, W. (2000, December). An efficient LDA algorithm for face recognition. In Proceedings of the International Conference on Automation, Robotics, and Computer Vision (ICARCV 2000) (pp. 34-47).

[15] Jain, U., Choudhary, K., Gupta, S. and Privadarsini, M. J. P. (2018, May). Analysis of Face Detection and Recognition Algorithms Using Viola Jones Algorithm with PCA and LDA. In 2018 2nd International Conference on Trends in Electronics and Informatics (ICOEI) (pp. 945-950). IEEE.

[16] Ahonen, T., Hadid, A. and Pietikainen, M. (2006). Face description with local binary patterns: Application to face recognition. IEEE transactions on pattern analysis and machine intelligence, 28(12): 2037-2041.

[17] Viola, P., \& Jones, M. (2001, December). Rapid object detection using a boosted cascade of simple features. In Proceedings of the 2001 IEEE computer society conference on computer vision and pattern recognition. CVPR 2001 (Vol. 1, pp. I-I). IEE.

[18] Ediz, Ç,. (2020). Adding Virtual Objects to Realtime Images; A Case Study in Augmented Reality. Sakarya University Journal of Computer and Information Sciences, 3(3), 188-200. 